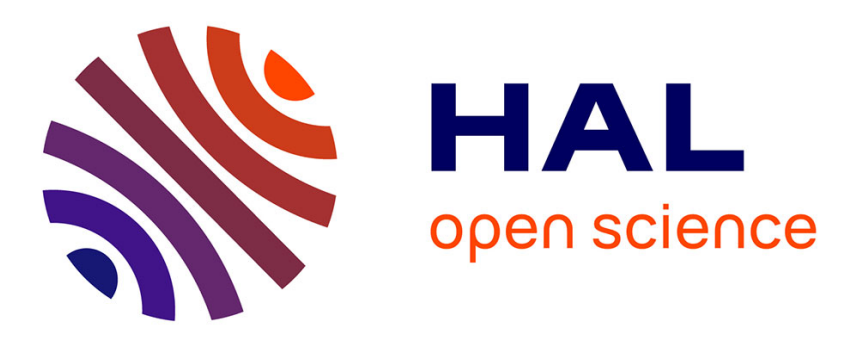

\title{
Error analysis for near-field EMC problems based on multipolar expansion approach
}

Zhao Li, François Tavernier, Arnaud Bréard, Laurent Krähenbühl, Damien Voyer, Carlos Sartori

\section{> To cite this version:}

Zhao Li, François Tavernier, Arnaud Bréard, Laurent Krähenbühl, Damien Voyer, et al.. Error analysis for near-field EMC problems based on multipolar expansion approach. IEEE Transactions on Magnetics, 2017, 53 (6), pp.1 - 4. 10.1109/TMAG.2017.2664985 . hal-01496540

\section{HAL Id: hal-01496540 \\ https://hal.science/hal-01496540}

Submitted on 26 Apr 2019

HAL is a multi-disciplinary open access archive for the deposit and dissemination of scientific research documents, whether they are published or not. The documents may come from teaching and research institutions in France or abroad, or from public or private research centers.
L'archive ouverte pluridisciplinaire HAL, est destinée au dépôt et à la diffusion de documents scientifiques de niveau recherche, publiés ou non, émanant des établissements d'enseignement et de recherche français ou étrangers, des laboratoires publics ou privés. 


\title{
Error analysis for near-field EMC problems based on multipolar expansion approach
}

\author{
Z. Li (李到) ${ }^{1}$, F. Tavernier ${ }^{1}$, A. Bréard ${ }^{1,3}$, L.Krähenbühl ${ }^{1,3}$, D. Voyer ${ }^{1,3}$ and C. A. F. Sartori ${ }^{2,3}$ \\ ${ }^{1}$ Université de Lyon - Ampère (CNRS UMR5005), ECL, 69134 Écully, France \\ 2 IPEN/CHEN-SP 05508-900 São Paulo, Brazil \& Escola Politécnica PEA/EPUSP, 05508-010, São Paulo-SP, Brazil \\ ${ }^{3}$ Laboratoire International franco-brésilien James-Clerk Maxwell (CNRS LIA817)
}

\begin{abstract}
Devices using power electronics are ubiquitous today and they are unfortunately intrinsic sources of electromagnetic interference. To address these electromagnetic compatibility problems at the initial design phase, a predictive method based on multipole expansion in spherical harmonics of the near field around each device was developed. To determine the basic expansions of a given source, a dedicated measurement bench has been designed. In this paper, some important issues of this approach and the measurement bench are studied, especially the error analysis on the measurements and the inverse problems. Some experimental results are also shown in the end.
\end{abstract}

Index Terms—Electromagnetic Compatibility, spherical harmonic, power electronics devices, quasi-static fields.

\section{INTRODUTION}

W ITH the advance of technology, electronic devices are moving in the direction of smaller size and increased power, which results in an increase of the electromagnetic interference (EMI) between two power electronic systems. These EMI may even cause malfunctions of the equipment. Hence, the issue of electromagnetic compatibility (EMC) becomes an essential activity in the conception of a new device. Conventionally, for the sake of the verification of the EMC norms, a large quantity of experimental tests is necessary after the production of a prototype. It causes additional costs and significant delays if the required standards are not reached. The origin of the problems is usually the magnetic nearfield coupling. This coupling can be between components (intra system coupling) and also between systems (inter system coupling). In order to solve this problem, a predictive method has been developed at Ampère Laboratory [1]. This method is based on the multipole expansion of the magnetic filed around the Device Under Test (DUT). The DUT can then be represented by an equivalent punctual source, which allows calculating the near-field coupling with other components.

A new automated measurement bench has been designed, dedicated to magnetic near-field measuring, which will be detailed later. Some related researches and experimental measurements will also be shown.

\section{MUltipole EXPANSION IN SPHERICAL HARMONICS}

A commonly used approach to determine the magnetic field development is to introduce the magnetic scalar potential $\psi$ :

$$
\mathbf{B}=-\mu_{0} \nabla \psi
$$

with $\mathbf{B}$ the magnetic induction field. In this work, the frequency range starts from $20 \mathrm{kHz}$ to $30 \mathrm{MHz}$. In this case, the quasi-static approximation is suitable because the size of the system is quite small compared to the wave length $(d<0.1 \lambda)$. Neglecting the displacement current and supposing that the wave only propagates in the air $(\sigma=0)$, the Laplace equation can be applied:

$$
\nabla \cdot \mathbf{B}=-\nabla^{2} \psi=0
$$

By separating the variables in spherical coordinates $(r, \theta, \varphi)$, the magnetic field for a point outside the sphere of validity (the minimal sphere which encloses the device) can be expressed in a multipole expansion [2]:

$$
\left.\mathbf{B}(r, \theta, \varphi)=\sum_{n=1}^{+\infty} \sum_{m=-n}^{+n}-\frac{\mu_{0}}{4 \pi} Q_{n m} \nabla\left(\frac{1}{r^{n+1}} Y_{n m}(\theta, \varphi)\right)\right)
$$

where $r$ is the distance from the origin of the expansion to the point where the field is expressed, $Q_{n m}$ are the coefficients characterizing the source, $Y_{n m}$ is the real spherical harmonic (SH) functions of $n^{t h}$ degree and $m^{t h}$ order given by [3].

Once the harmonic coefficients of the two systems are identified, the inductive coupling between them can be determined through the calculation of the mutual inductance:

$$
M_{A B}=\frac{1}{\mu_{0} I_{A} I_{B}} \sum_{n=1}^{+\infty} \sum_{m=-n}^{+n}(-1)^{m}\left({ }_{A} Q_{1 n,-m} \cdot{ }_{B} Q_{1 n m}\right)
$$

where $I_{A}$ and $I_{B}$ are the currents circulating in the source A and B. It is important that the two developments in SH have the same origin, which can be achieved by the Addition Theorem for Spherical Harmonics [4]. The accuracy of the source representation and mutual inductance calculation increase with $N_{\max }$. The truncation of the terms higher than $N_{\max }$ will necessarily engender a bias between the measurement and the reconstructed model. Thus, the choice of $N_{\max }$ needs a lot of prudence, which will be shown in VI.

\section{Presentation of the new automated MEASUREMENT BENCH}

A new automated measurement bench was developed at Ampère laboratory. This bench allows two rotational movements via: a PVC arm in the vertical direction $(\theta)$ and a source support in the horizontal direction $(\varphi)$ as shown in Fig. 1. 


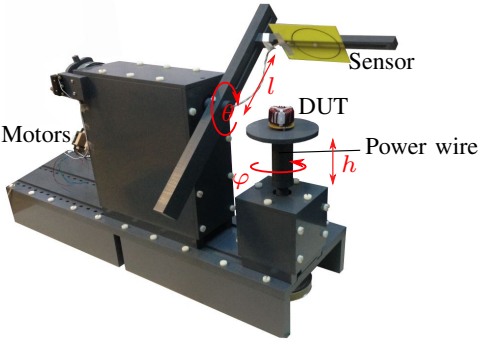

Fig. 1. Automated measurement bench

These rotations are controlled by a JAVA program and realized by two stepper motors and pulleys which are dedicated to avoid the parasitic influence. The DUT is placed at the center and is supplied by a Vector Network Analyzer (VNA). The voltage ratio between the sensor and the DUT is measured. The measurement bench is surrounded by the electromagnetic absorber, which allows to reduce the environmental noise. A set of measurements can be done with the measuring positions uniformly distributed on a spherical surface. The radiation of the DUT can then be identified and expressed in multipole expansion. Meanwhile, the length of the measuring arm $(l)$ and the dimension of the magnetic flux sensor can both be changed according to the DUT size. These two parameters have a direct influence on the calculation accuracy, as it will be detailed in the followed section. Due to mechanic restrictions, the vertical measuring range $(\theta)$ is limited between $-120^{\circ}$ and $120^{\circ}$.

\section{THE OPTIMAL DIMENSION OF THE SENSOR}

In the previous work, a measurement bench has been developed which is based on spatial filtering [5]. Therefore, this bench was limited to the fourth degree $\left(N_{\max }=4\right)$ and a large quantity of measurements is necessary to identify the non-zero-order component. As an inheritance of the former bench, we chose a circular coil as the sensor to measure the magnetic flux. The question now is how to choose the size of the coil. Ideally, the sensor dimension has no influence on the identification of the DUT. However, the uncertainties must be considered in experimental measurements. In this research, the involved uncertainties consist: sensor position $\left(\approx 0.1^{\circ}\right)$, DUT position $(\approx 2 \mathrm{~mm})$, DUT orientation $\left(\approx 0.5^{\circ}\right)$, arm length $(\approx 5 \mathrm{~mm})$ and the environmental noise $\left(\approx 10^{-10} \mathrm{~Wb}\right)$. The environmental noise is characterized by the measurement without sources, which is considered as a white noise. An academic example with several vertical coils is studied, which is actually the assemblage of the canonical sources of the first four degrees. Thanks to the axi-symmetry, the identification of the DUT needs only the vertical measurements. With all the mentioned uncertainties, a large quantity of samples were achieved for each sensor size. The sensors are always placed $20 \mathrm{~cm}$ away from the DUT center in the first place. The corrected sample standard deviation is calculated for each harmonic component (Fig. 2) as a function of the radius of the sensor. In order to make the comparisons more visible, the deviations are all normalized to the same scale. It is noted that the deviations decrease at the beginning between $1 \mathrm{~cm}$ to $6 \mathrm{~cm}$. Because the more the sensor size increases, the more

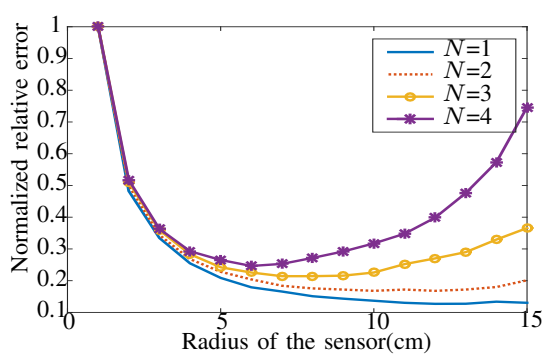

Fig. 2. Normalized sample standard deviations of each $\mathrm{SH}$ component as a function of the sensor dimension

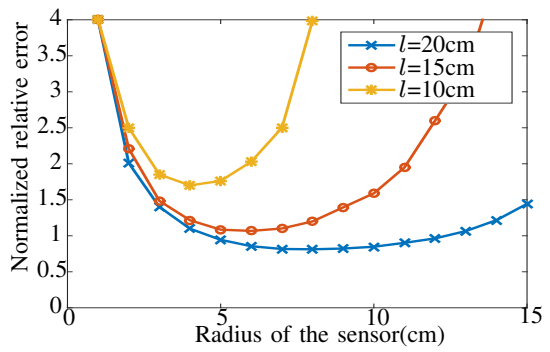

Fig. 3. The sum of the normalized sample standard deviation as a function of the sensor dimension

magnetic flux will be captured, the more the signal-to-noiseratio will be important. However, the sensor size determines also the sphere of measurement. There is a risk of losing information of high orders when the sphere of measurement is too large. For this reason, the deviations on the components of third and fourth degree begin to increase from $8 \mathrm{~cm}$. In order to find a compromise between the signal-to-noise-ratio and the information of high degrees, the summation of all the normalized standard deviations of the first four degrees is shown in Fig. 3. It is worth noting that the sums of the deviations are in the form of a parabola, which possesses a minimum for a given sensor size. We can also see that the closer the measurement is $(l=15$ or $10 \mathrm{~cm})$, the more we need to reduce the size of the sensor. In fact, when the sensor is closer to the DUT, the quantity of the captured magnetic flux increases, so the influence of the sensor size on the signal-tonoise-ratio will be less important. From here on, in this paper, the radius of the sensor is fixed to $5 \mathrm{~cm}$.

\section{ITERATIVE NORMALIZATION TO DECREASE THE CONDITION NUMBER}

Once a set of measurements is done for a DUT, the identification is possible. The problem is a linear system which can be expressed in the matrix form:

$$
\mathbf{A} \cdot \mathbf{X}=\mathbf{B}
$$

where A contains the SH functions, $\mathbf{B}$ represents the measurements of the magnetic flux and $\mathbf{X}$ contains the SH coefficients of the DUT to be identified. There are a lot of methods to invert the system and find $\mathbf{X}_{\mathbf{0}}$, which is the nearest solution to the measurement. The condition number, which is the ratio between the largest and the smallest singular value of $\mathbf{A}$, 
represents the sensibility of the system. Taking the positions of measurement shown in Fig. 4 as an example, the obtained condition number is $3 \cdot 10^{5}$, making the system ill-conditioned. On the one hand, it means that the output value of the system could vary enormously with a small change in the input argument. On the other hand, it means that among all the measurements, some carry no information in the calculation. That is why the condition number is a very important property for a linear system.

There exist many methods of regularization to reduce the condition number of the ill-conditioned system. However, the effects are sometimes not sufficient because the value of each row is not taken into account in calculating the norm of each column. The natural idea is to normalize the lines after the columns. However, the normalization of the lines inevitably degrades those of the columns and vice versa. This is why an iterative process is used, which normalizes columns and lines until the condition number of the matrix stabilizes to its minimum. This method is frequently used to obtain better conditioned linear systems[6][7].

Each iteration consists of finding respectively two new matrices of normalization of columns and rows: $\mathbf{W}_{c}^{(i)}$ and $\mathbf{W}_{l}^{(i)}$. After the $i^{t h}$ iteration, the solution of $\mathbf{X}$ is given by [6]:

$$
\mathbf{X}_{\mathbf{0}}=\prod_{i} \mathbf{W}_{c}^{(i)} \cdot\left[\prod_{i} \mathbf{W}_{l}^{(i)} \cdot \mathbf{A} \cdot \prod_{i} \mathbf{W}_{l}^{(i)}\right]^{+} \cdot \prod_{i} \mathbf{W}_{c}^{(i)} \cdot \mathbf{B}
$$

where ${ }^{+}$represents the generalized inverse. This algorithm shows fast linear convergence with an asymptotic rate of about 0.5 . The condition number converges and is reduced to about 7 after 10 iterations. At this point, our system is well-conditioned and thus much more insensitive to the noises and measurement uncertainties.

\section{THE RESIDUAL OF THE MEASUREMENT}

The residual in the least squares method represents the bias of the model estimation compared to the measurements, which could be a criterion of the estimation quality [8]. The vector of residual is defined by:

$$
\mathbf{R}=\mathbf{A} \cdot \mathbf{X}_{\mathbf{0}}-\mathbf{B}
$$

$\mathbf{R}$ is a random variable with expected value of zero and variance $E\left\{\mathbf{R} \cdot \mathbf{R}^{t}\right\}$, which gives the distribution of the deviation between the model and the measurements and is an important statistical tool for system diagnosis. On the one hand, it is easier to detect and eliminate erroneous measurements, for example when the residuals of some measurements are much more important than others. On the other hand, the residual can help to determine the maximal degree $N_{\max }$ for a multipole expansion. Let us take a coil with a radius of $5 \mathrm{~cm}$ as an example. Due to the axi-symmetry of the source, only the zeroorder components exist. 25 vertical measurements from $0^{\circ}$ to $120^{\circ}$ are performed at a distance of $10 \mathrm{~cm}$ from the DUT. The sensor is always orthogonal to the spherical surface of measurement. In Fig. 5, the different curves are the estimated model with different maximal degree $N_{\max }$. It is noted that as $N_{\max }$ increases, the curve fits better and better to the

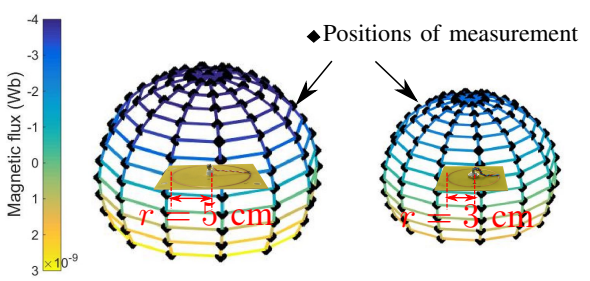

Fig. 4. The measured magnetic flux around two circular coils with different sizes, the black points represent the positions of measurement.

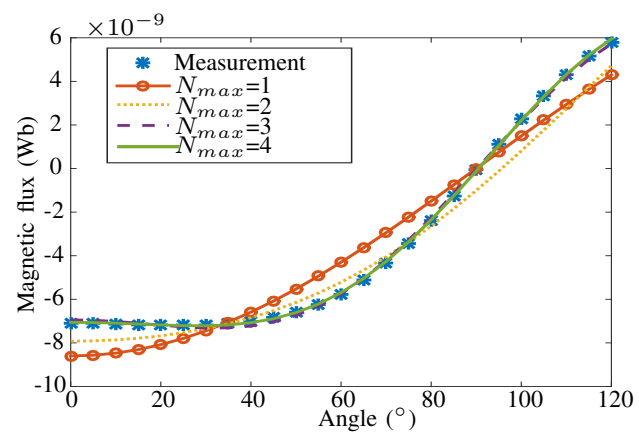

Fig. 5. The measurements of the magnetic flux and the estimated model reconstructed by $\mathrm{SH}$ with different degrees

measurements and they practically superpose when $N_{\max }=4$. However, this visual method is not accurate and it becomes unpractical when the non-zero-order components exist with the spatial measurements. This is why the introduction of the residual is so significant.

In Table I, the standard deviation of the residual is shown as a function of $N_{\max }$. The standard deviation decreases with $N_{\max }$ and tends to a constant. It has been mentioned in section III that the environmental noise is a white noise with an magnitude order of $10^{-10} \mathrm{~Wb}$. Hence, it is meaningless to continue to increase $N_{\max }$ if the standard deviation of the residual is already smaller than the noise. Indeed, if the standard deviation of the residual is much smaller than the environmental noise, there could be deviations in the high degree SH components. In the case of the circular coil, $N_{\max }=4$ is appropriate for the identification.

\section{EXPERIMENTAL RESULTS}

In order to test and verify the measuring bench, two printed circular coils with radius of 5 and $3 \mathrm{~cm}$ are identified in spherical harmonics (Fig. 4) with the shown positions of measurement. The mutual inductance between them along different directions is also calculated and compared to the analytical formulas [9]. The measured magnetic flux is shown, which proves the axi-symmetry of the two coils. However, to ensure that the power wires are taken into account, the DUTs are identified with all-order components by the approaches of section V. Afterwards, by applying equation (4), the mutual inductance between the two coils can be calculated with respect to their relative position. In the Fig. 6, the coils are positioned respectively vertical and horizontal. In both situations, the mutual inductance is obtained in three ways: analytical, direct 
TABLE I

\begin{tabular}{c||c|c|c|c|c|c}
\hline $\mathbf{N}_{\max }$ & 1 & 2 & 3 & 4 & 5 & 6 \\
\hline Standard deviation of the residual & $1.1 \cdot 10^{-9}$ & $7.1 \cdot 10^{-10}$ & $1.2 \cdot 10^{-10}$ & $5.9 \cdot 10^{-11}$ & $1.3 \cdot 10^{-11}$ & $1.1 \cdot 10^{-11}$ \\
\hline
\end{tabular}
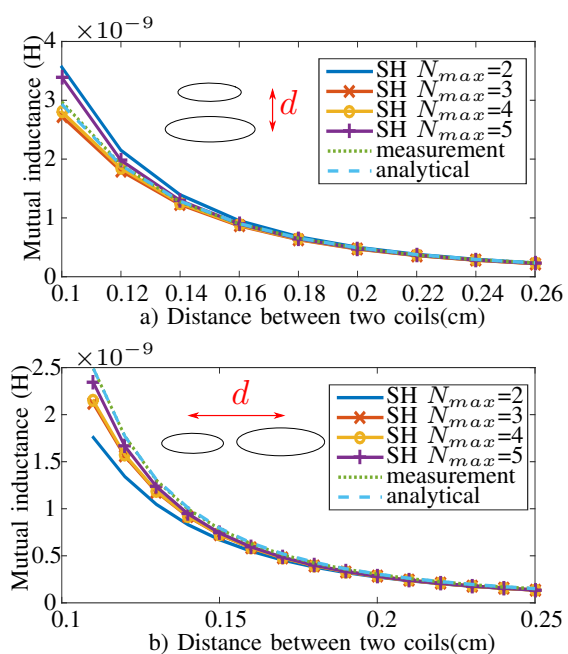

Fig. 6. The mutual inductance between two circular coils as a function of the distance between them: a) vertical; b) horizontal

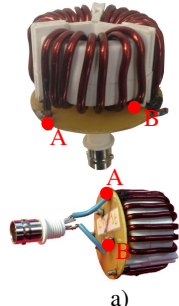

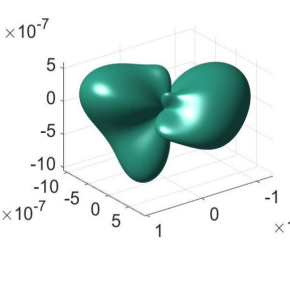

b)

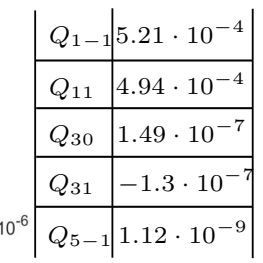

c)
Fig. 7. a) the toroid inductor, one-third is powered between $\mathrm{A}$ and $\mathrm{B}$; b) its magnetic induction $(\mathrm{T})$; c) primary $\mathrm{SH}$ components of this toroid inductor

measurement and $\mathrm{SH}$ approach. It is noted that the direct measurements correspond perfectly to the analytical results and the accuracy of the SH approach increases with the maximal degree considered $\left(N_{\max }\right)$. When $N_{\max }=4$, the three curves almost superpose and the relative error between the analytical and the $\mathrm{SH}$ approach is much lower than former results obtained in [1]. However, exceptions still exist such as $N_{\max }=5$ for the vertical case a). The accuracy is worse than $N_{\max }=4$ when the two sources are very close to each other. Indeed the fifth degree is affected by the environmental noise to a great extent in minimizing the standard deviation as mentioned in section VI. And the high degree SH components account for a large proportion in the near-field computation because of the term of $r^{n+1}$ in equation (3). Consequently, the mutual inductance with $N_{\max }=5$ is less accurate than $N_{\max }=4$ when the coils are very close. The process is strictly the same for the identification of the complex sources, such as a toroid inductor as shown in the Fig. 7a). Only a third part of the toroid inductor is powered. After the measurements all around the DUT, it is noted that for reducing the standard deviation of the residual until necessary (smaller than $10^{-10}$
$\mathrm{Wb}), N_{\max }$ must be equal to or greater than 5 . The magnetic radiation $5 \mathrm{~cm}$ away from its center is also shown in Fig. 7b), which is reconstructed by its $\mathrm{SH}$ components as shown in c). With these $\mathrm{SH}$ components, the mutual inductance between this toroid inductor and any other devices can be computed in terms of their relative position.

\section{CONCLUSION AND PERSPECTIVE}

In this paper, a new automated bench is presented, which is based on the measurement of magnetic near-field. The multipole expansion is applied allowing not only the identification of any radiation source, but also the calculation of the inductive couplings with other devices. By studying the accuracy of the bench, the optimal dimension of the sensor is deduced and the introduction of the residual can help us to choose the $N_{\max }$ according to the environmental noise. In the end, some experimental results are shown, including the mutual inductance between two printed circular coils and the identification of a toroid inductor. Due to the $r^{n+1}$ term in equation (3), in the measurement, it is noticed that when the sensor is further from the DUT, the accuracy of the low degree SH components is better than the high degree and it is contrary when the sensor is closer. Our future work is to take advantage of this propriety by measuring the DUT by several steps with different arm lengths. The Bayesian method could be applied to insert the results of the previous measurements to the new measurements as a prior information. The prior information could also be the knowledge of the geometry of the DUT or mechanic uncertainties in the measurements [10].

This work is supported by the French Agence Nationale de la Recherche (PolHar-CEM project : ANR-14-CE22-0009).

\section{REFERENCES}

[1] S. Zangui, "Détermination et modélisation du couplage en champ proche magnétique entre systemes complexes," Ph.D ECL, 2011.

[2] J.C.Nédélec, "Acoustic and electromagnetic equations integral representations for harmonic problems," Springer, 318 pages, 2001.

[3] T. Q. V. Hoang, A. Bréard, and C. Vollaire, "Near magnetic field coupling prediction using equivalent spherical harmonic sources," IEEE Transactions on EMC, vol. 56, no. 6, pp. 1457-1465, Dec 2014.

[4] B. C. Brock, "Using vector spherical harmonics to compute antenna mutual impedance from measured or computed fields," SANDIA, 2001.

[5] B. Vincent, O. Chadebec, J.-L. Schanen, K. Berger, R. Perrussel, and L. Krähenbühl, "Identification of equivalent multipolar electromagnetic sources by spatial filtering," IEEE Transactions on Magnetics, vol. 46, no. 8, pp. $2815-2818$, Jul. 2010.

[6] D.Ruiz, "A scaling algorithm to equilibrate both rows and columns norms in matrices," ENSEEIHT-IRIT Technical Report, 2001.

[7] J.Francklin, "On the scaling of multidimensionnal matrices," Linear Algebra and its applications, 1989.

[8] L. Schmerber, "Identification et caractérisation de sources électromagnétiques - application à la discrétion des moteurs de propulsion navale," Ph.D. dissertation, INP, 2006.

[9] E.Durand, Magnetostatique. Masson et Cie Editeurs, 1968.

[10] L. Schmerber, L. L. Rouve, and A. Foggia, "Spherical harmonic identification using a priori information about an electrical device," IEEE Transactions on Magnetics, vol. 43, no. 4, pp. 1781-1784, April 2007. 